\title{
Wavelet analysis of long-term variations in neutron monitor, sunspot number and interplanetary magnetic field data
}

\author{
Fraser Baird', Alexander MacKinnon ${ }^{2}$ \\ Correspondence \\ 'University of Surrey, Guildford, UK, f.baird@surrey.ac.uk \\ ${ }^{2}$ School of Physics and Astronomy, University of Glasgow, UK, alexander.mackinnon@glasgow.ac.uk
}

\section{OPEN ACCESS}

This work is published under the Creative Commons Attribution 4.0 International license (CC BY 4.0) Please note that individual, appropriately marked parts of the work may be excluded from the license may be excluded from the license mention other copyright conditions. If such third party material is no under the Creative Commons license any copying, editing or public repro duction is only permitted with the prior consent of the respective copyright owner or on the basis of relevant legal authorization regulations.

\section{Keywords}

neutron monitors; wavelets; solar activity

\begin{abstract}
We use wavelet analysis to investigate the characteristic timescales appearing in neutron monitor data and their solar drivers. Wavelet analysis of Oulu NM data shows the well-known roughly 11 -year variation in intensity, but with a period contracting during solar cycle 22. This is also seen in the wavelet analysis of the interplanetary magnetic field magnitude data. However, this varying period is not shown in the wavelet analysis of daily estimated sunspot numbers, a proxy for solar magnetic field strength. The time at which the wavelet transform is found to be similar for both NM and sunspot number, but not for the interplanetary magnetic field magnitude. This further highlights a complex relationship between cosmic rays, the heliosphere, and Earth.
\end{abstract}

\section{Introduction}

The time series produced by the count rate in a Neutron Monitor (NM) contains information on the variation of cosmic ray (CR) intensity, above the appropriate cutoff rigidity, arriving at Earth. The CR flux at Earth in turn is modulated by solar magnetic activity and its effect on the magnetosphere. When analysed using Fourier series methods, NM time series show persistent periodic behaviour on scales recognisable from the Sun: the solar rotation period; the 11-year activity cycle; and other periods found in sunspot number (e.g. Kudela et al. 1991).

Fourier methods are ideal for identifying persistent periodic behaviour in a time series. Wavelet methods (Farge 1992; Torrence \& Compo 1998) go further, providing a decomposition of a time series in which the amplitude at a fixed period can vary with time. Variations of amplitude will contain information on the internal dynamics of the system being studied as well as its external drivers.

The variable appearance of a period in NM data prompts obvious questions. Do periods in NM data solely mirror the solar drivers, or is there also a component from the response of the terrestrial magnetosphere? Here we begin an analysis of such questions by comparing the time behaviour of the power at particular periods, obtained from wavelet analysis, comparing this behaviour with measures of solar magnetism, starting with sunspot number, and of the state of the solar wind at Earth. We start by presenting key wavelet ideas, before presenting wavelet transformations of NM, sunspot data and Interplanetary Magnetic Field (IMF) measurements. Differences and similarities are revealed by this process, which we discuss. 


\section{Method}

The wavelet transform is defined as the convolution of a time series with a wavelet function, dilated to a scale and translated to a time:

$$
W(s, \tau)=\sum_{\mathrm{t}=0}^{\mathrm{N}-1} T(t) \psi_{s}^{*}(t-\tau)
$$

Where $W(s, \tau)=$ wavelet transform

$T(t)=$ discrete time series with $N$ data points, $\{t \in \mathbb{Z} \mid 0 \leq t<N\}$

$\psi_{s}^{*}=$ complex conjugate of the wavelet function (see eq. 2 )

$s=$ scale to which the wavelet function is dilated, analogous to the period of oscillation.

In general, a wavelet function must satisfy the following conditions: be of zero mean, and be localised in time and frequency domains. For this study, the wavelet function utilised was the second derivative of a complex gaussian function:

$$
\psi_{\mathrm{s}}(t)=\frac{\mathrm{C}}{\sqrt{s}}\left(4 t^{2}+4 i t-3\right) e^{-\left(t^{2}+i t\right)}
$$

As with Fourier transforms, discontinuities in the time series cause large peaks in the wavelet transform. While this can be helpful if one wishes to identify discontinuities in the signal, the end points of the time series create artefacts near the end points of the wavelet transform. In order to screen out these edge effects, we employ a cone of influence (COI). The COI defines a minimum and maximum time index depending on scale, and values outwith the range defined by the COI are set to zero, i.e.

$$
W_{\text {COI }}(s, \tau)=\left\{\begin{array}{lc}
W(s, \tau), \tau_{\text {min }}(s)<\tau<\tau_{\max }(s) \\
0, & \text { otherwise }
\end{array}\right.
$$

For the wavelet function shown in eq. 2 the minimum and maximum values are given as

$$
\tau_{\min }(s)=\sqrt{s}, \quad \tau_{\max }=\tau_{N-1}-\sqrt{s}
$$

Data were obtained for the interval 1964/04/01 to 2020/06/30 for three quantities: Oulu NM count rate, IMF magnitude, and estimated daily sunspot number (SSN). For each of these data the wavelet transform was computed using eq. 1 at a range of scales, $s=[1,4096$ ], corresponding to periods $T=6$ days, 28 years. This was performed using the PyWavelets implementation of the continuous wavelet transformation (Lee et al. 2019). The COI was applied, and the result of each of these transformations was then squared, and plotted as a scalogram. Note that prior to taking the transformation, the IMF data was filtered with a 10-day running mean, so the very first scale does not yield a physical result. 


\section{Results}

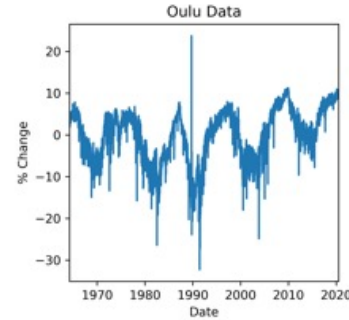

Oulu Wavelet Transform

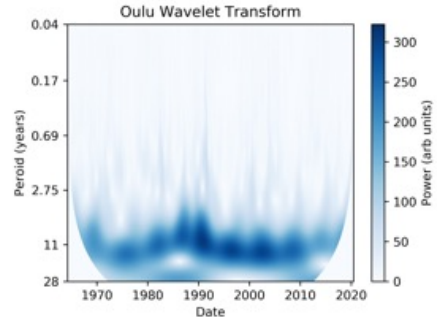

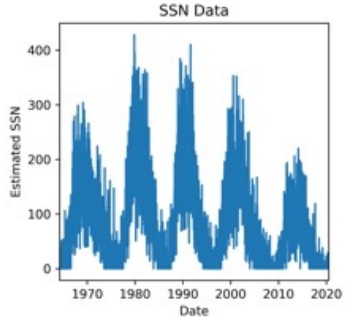

SSN Wavelet Transform

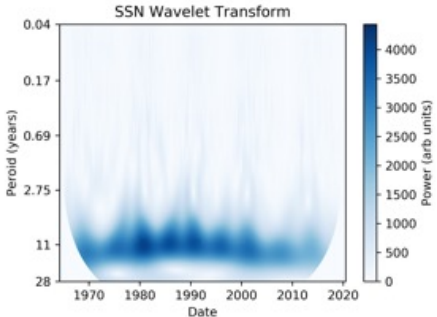

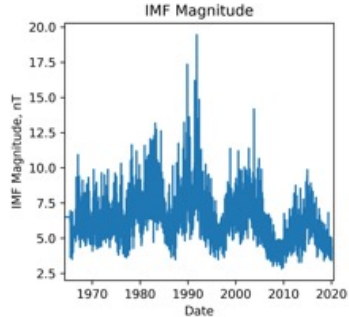

IMF Wavelet Transform

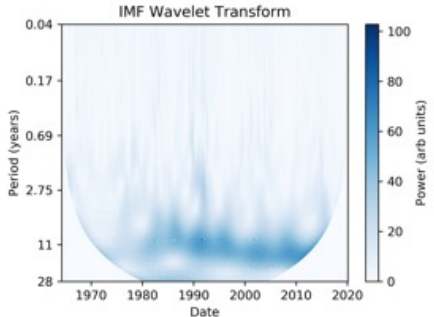

Figure 1: Data (top row) and their WT scalograms (bottom row) for Oulu NM (left column), SSN (middle column) and IMF Magnitude (right column).

The input data and resulting scalogram for all quantities are presented in figure 1. Note that the y-axis of the scalogram is a log scale. It is evident from the scalograms that each data exhibits a consistent $\sim 11$ yr variation. While the SSN scalogram shows that the period of the variation is fairly constant, this is not mirrored in the NM or IMF scalograms. The NM scalogram clearly shows a contraction in the period of variation between 1980 and 1990, before returning to a period closer to 11 years in the following decades. This behaviour is also seen in the IMF scalogram, which is of poor resolution.

While the SSN and NM scalograms differ in the constancy of period, they both show similar trends in the power of the scalogram, though they do not match exactly. While both scalograms show low power of variation from 2000 to 2020 (particularly the latter decade), the maximum power occurs around 1980 for the SSN and 1990 for the NM. Interestingly, the IMF scalogram differs completely in terms of power. The time of maximum power occurs between 2000 and 2020, and the minimum between 1970 and 1980 .

\section{Discussion and conclusion}

As noted in the previous section, the IMF magnitude scalogram is of low quality. This is likely due to the intermittent nature of this data; since it is provided by different satellites over time, gaps in the data are inevitable due to operational challenges and periods between missions. While these gaps have been filled in with linearly interpolated values, the gaps still create sections of the data where absolutely no periodic variations are present.

It is not particularly surprising that the SSN scalogram doesn't perfectly match that of the NM or IMF magnitude - sunspots are only a proxy for solar magnetic activity and other factors will play a role in the CR activity at Earth. However, the magnitude of the difference is unexpected.

Since the strength of the IMF is associated with the modulation of cosmic rays (Beer et al. 2012), it follows that the long-term variations of the IMF should be mirrored in that of the NM data. The changing period of the long-term variation is similar in both scalograms, but the power of the variation is not similar in either scalogram. It could be that the aforementioned data gaps are the main cause of the IMF transform power. 
From this study, a number of conclusions can be drawn. Firstly, that wavelet analysis has also identified the well-known persistent, long-term, periodic variation of $\sim 11$ years in NM, SSN, and IMF magnitude data. Secondly, that the period of this variation contracts between 1980 and 1990 in the NM and IMF data, but not the SSN data. Thirdly, the time of maximum and minimum power of the NM and SSN scalograms are well matched, however the IMF scalogram does not match the other two in this regard. The physical origin of these differences will be investigated further elsewhere, together with the co-variability of NM and solar measurements at other timescales.

\section{Acknowledgments}

We thank the NMDB database (www.nmdb.eu) for providing data and the Sodankyla Geophysical Observatory (http://cosmicrays.oulu.fi/) for the Oulu NM data. We also thank WDC-SILSO, Royal Observatory of Brussels, Belgium (www.sidc.be/silso/) for providing SSN data, and NASA/GSFC Space Physics Data Facility‘s OMNIWeb service (https://omniweb.gsfc.nasa.gov/index.html) for provision of IMF data.

\section{References}

Beer, J., McCracken, K., von Steiger, R., 2012, Cosmogenic Radionuclides, Springer, DOI: https://dx.doi.org/10.1007/978-3-642-14651-0_5 Farge, N., 1992, Ann. Rev. Fluid Mech. 24, 395, DOI: https://dx.doi.org/10.1146/annurev.fl.24.010192.002143

Lee, G. R. , Gommers, R. ,Wasilewski, F., Wohlfahrt, K., O'Leary, A., 2019, Journal of Open Source Software, 4, 36, 1237, DOI: https://dx.doi. org/10.21105/joss.01237

Torrence, C., Compo, G. P., 1998, Bull. Amer. Meteor. Soc. 79, 61, DOI: https://doi.org/10.1175/1520-0477(1998)079\%3C0061:APGTWA\%3E2.0.CO;2

Kudela, K., Ananth, A. G., Venkatesan, D., 1991, JGR 96, 15871 DOI: https://dx.doi.org/10.1029/91JA01166 